\title{
Can the science of well-being be objective?
}

Forthcoming in The British Journal for the Philosophy of Science

\section{Anna Alexandrova}

Abstract: Well-being, health and freedom are some of the many phenomena of interest to science whose definition relies on a normative standard. Empirical generalizations about them thus present a special case of value-ladenness. I propose the notion of a 'mixed claim' to denote such generalizations. Against the prevailing wisdom, I argue that we should not seek to eliminate them from science. Rather, we need to develop principles for their legitimate use.

Philosophers of science have already reconciled values with objectivity in several ways, but none of the existing proposals are suitable for mixed claims. Using the example of the science of well-being, I articulate a conception of objectivity for this science and for mixed claims in general.

\section{Table of Contents}

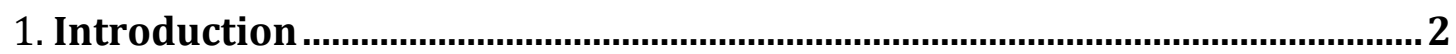

2. What Are Mixed Claims? ................................................................................ 3

3. Mixed Claims Are Different.................................................................................. 5

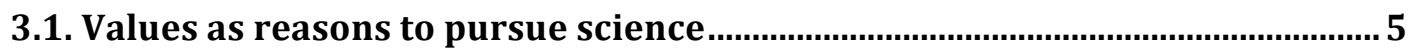

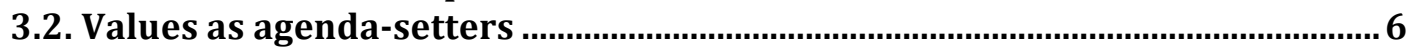

3.3. Values as ethical constraints on research protocols........................................... 6

3.4. Values as arbiters between underdetermined theories .................................... 6

3.5. Values as determinants of standards of confirmation ........................................ 7

3.6. Values as sources of wishful thinking and fraud ................................................ 7

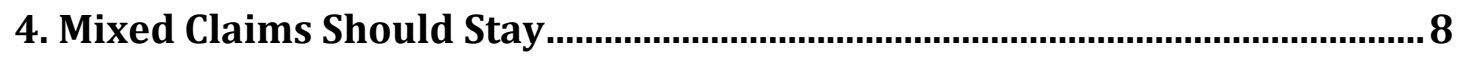

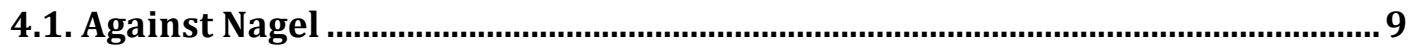

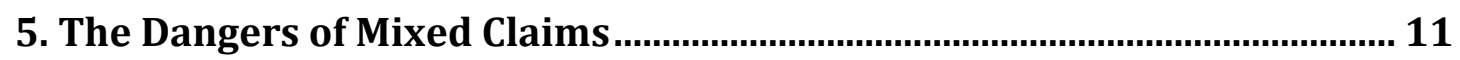

6. The Existing Accounts of Objectivity .......................................................... 12

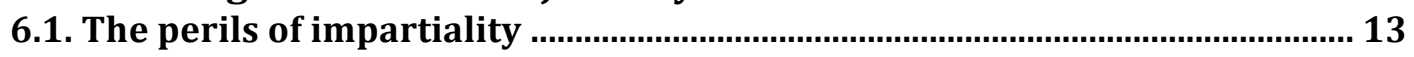

7. Objectivity For Mixed Claims ...................................................................... 14

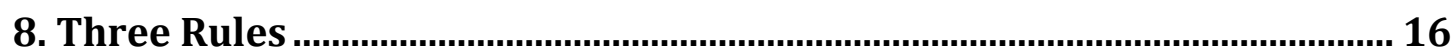

8.1 Unearth the value presuppositions in methods and measures......................... 16

8.2 Check if value presuppositions are invariant to disagreements. .................... 17

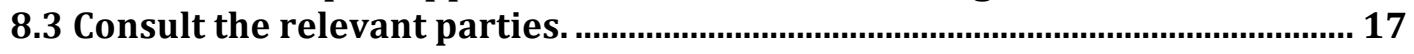

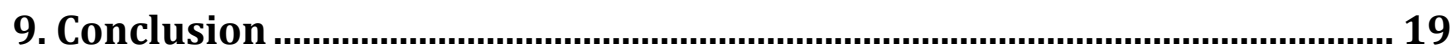




\section{Introduction}

Consider a claim ' $C$ causes $E$ under conditions $N$ ' that is well confirmed by the lights of the scientific discipline in which this claim figures. What if the definition of $\mathrm{C}, \mathrm{E}$, or $\mathrm{N}$ presupposed a moral standard that in turn determined how $\mathrm{C}, \mathrm{E}$, or $\mathrm{N}$ are conceptualized and measured? Would you trust this claim? Would you grant it objectivity? Would you let it be part of science at all?

Empirical claims about health, well-being, child development, freedom, economic growth, resilience, frailty and so on, appear to have such a structure. They relate ordinary purely empirical variables, such as geographic location, with a variable that is defined in partly normative terms, such as health status, as in 'Living in East Saint Louis harms health'. Or they may relate two variables that both appear to have a normative component as in 'Unemployment negatively impacts wellbeing'. 'Health' and 'unemployment' as concepts are partly normative in the sense that their definition and measurement, at least on the face of it, depend on normative judgments about what it takes to be healthy and what it takes to be involuntarily out of work. I propose to call causal or correlational claims with such normative presuppositions 'mixed claims' because they mix the normative and the empirical in a way that ordinary scientific claims do not. Mixed claims typically occur in social and medical sciences such as economics, and clinical and developmental psychology, but they can also be found in the biological and environmental sciences too.

The problem is that the typical conception of scientific objectivity has not caught up with this reality. Although philosophers have noted instances of mixedness, there is no clarity on what to do about them. Objectivity of science understood as value-freedom has been a dominant conception in twentieth century philosophy of science and would seem to counsel against mixed claims. But this conception is slowly losing its grip as values acquire a legitimate place in science. Yet even as the layers of value-freedom are being peeled off, there is still no positive story about how projects that rely on mixed claims can be both value-laden and objective.

In this paper I argue in favor of the following:

1) Mixed claims are distinct from other well-rehearsed ways in which science can be value-laden. (Sections 2 and 3)

2) Some claims in the social and medical sciences should remain mixed, against the advice to reformulate them into value-free claims or to move them outside science. (Section 4)

3) The existing accounts of objectivity that make room for values do not fit mixed claims. (Sections 5 and 6 )

4) Nevertheless mixed claims can be objective in a sense that I articulate and defend. (Sections 7 and 8)

I couch these theses as concerning mixed claims in general, but my examples will be mostly drawn from the science of well-being. It is worth concentrating on well-being for two reasons. First, it is arguably the most prominent of the recent mixed projects in science. It is studied all across social, psychological and 
medical sciences, has a number of newly dedicated journals and professional societies, and regularly tops the lists of the most popular key words in abstracts of published papers. ${ }^{1}$ The second and best reason to focus on well-being is that other normative concepts regularly bottom out in well-being. Measures of health, growth, development etc. are justified in part on the basis of their ability to capture well-being. ${ }^{2}$ So an account of how the science of well-being can be objective will take us a long way towards understanding mixed claims in general.

\section{What are mixed claims?}

'Happiness is not always conducive to well-being' (Grueber et al [2011])

'Long commutes are associated with lower well-being' (Diener et al [2008])

'Early learning difficulties have a disproportionate impact on life well-being' (Beddington et al [2008])

Social scientists who make such claims rely on a conception of well-being. In psychology this conception is one of the following three. The first one, a revival of hedonism, treats well-being as happiness or a favorable balance of positive over negative emotions (Kahneman et al [2004b]). This can be measured by various experience sampling methods. The second tradition takes well-being to consist in life satisfaction, an individual judgment about how one's life is doing overall (Diener et al [2008]). The main measurement tool here is a self reported judgment of life satisfaction. Finally, a third approach speaks of well-being as flourishing or good functioning, an ensemble of strengths such as competence, relatedness, sense of achievement and meaning, measured by self-reports about these aspects of life (Ryan and Deci [2001]). Settling on one of these approaches appears to be a choice about the most plausible conception of well-being. This normative step is important because one of the goals of the psychology of wellbeing is to understand whether and which positive emotions are good for us: how they enable better functioning of individuals and communities, but also whether they sometimes harm us. ${ }^{3}$

So I propose a definition:

A hypothesis is mixed if and only if:

1. It is an empirical hypothesis about a putative causal or statistical relation.

\footnotetext{
${ }^{1}$ See Zacks and Malley [2007], as well as Journal of Happiness Studies, International Journal of Wellbeing, and the International Society for Quality of Life Studies.

2 The World Health Organization's 1946 definition of health is 'a state of complete physical, mental and social wellbeing and not merely the absence of disease or infirmity'(World Health Organization [1948]).

3 For example Fredrickson [2001] and Grueber et al [2011].
} 
2. At least one of the variables in this hypothesis is defined in a way that presupposes a moral, prudential, political or aesthetic value judgment about the nature of this variable.

The first part of this definition specifies mixed hypotheses as the causal or correlational claims ${ }^{4}$ typical in social and medical science. (They are normally probabilistic hypotheses relating two or more kinds). Such claims play a crucial role in explanations and policy interventions. But I phrase my definition in terms of causal claims only for simplicity. They do not exhaust the science of well-being (or any other science for that matter). Causal claims are not necessarily more fundamental or more important than theoretical claims, non-propositional knowledge, images, instruments etc. So we can equally well have mixed theories, mixed measures and more generally mixed sciences. In this paper I consider mixed hypotheses only but nothing, I believe, rides on this choice.

The more crucial feature of mixed claims is in the second part of the definition, that is their reliance on a normative judgment. Such a reliance occurs in two ways. First, a scientist might adopt a given measure because she believes it reflects well-being better than other measures - an explicit normative judgment. Secondly, a scientist might follow a set procedure for measurement or data collection - for example, she might collect data on reported satisfaction with life - but this procedure is part of a broad methodological decision adopted by the founders of the research program of which the scientist is a member. In this case, adoption of a measure betrays an implicit normative commitment to the validity of this research program. Either way the outcomes of the process are mixed claims, whether explicitly or implicitly.

What sort of values make for mixed claims? Philosophers distinguish between cognitive values - simplicity, explanatory power, coherence, generality etc. - on the one hand, and non-cognitive values - moral, prudential, political or aesthetic - on the other (Longino [1990], Lacey [2005]). It is the second kind that figures in mixed claims. For science of well-being (and also plausibly health and child development sciences) the most relevant values are prudential. In other cases, such as claims about involuntary employment, dignity at work, or political legitimacy, the values presupposed are ethical and political.

Without identifying them as mixed claims, philosophers have noted normative content in the scientific study of efficiency (Nagel [1961], Hausman and McPherson 2006]), rape (Dupre [2007]), spousal abuse (Root [2007]), unemployment (Hausman and McPherson [2006]), divorce (Anderson [2004]), inflation (Reiss [2010]), aggression (Longino [2013]), AHDH (Hawthorne [2013]) and, of course, well-being (Tiberius [2004]). My notion of a mixed claim captures these examples. The task is to settle whether mixed claims should be part of science and if so what rules they should obey.

This focus should be distinguished from the broader project of understanding the nature and significance of 'thick concepts'. Ever since Bernard Williams

\footnotetext{
${ }^{4}$ Here I use 'claim' and 'hypothesis' interchangeably.
} 
coined this expression philosophers have referred to 'well-being', 'courage', 'kindness', 'care' etc as thick, differentiating them from 'good' and 'right' which are supposedly 'thin'. Although the precise definition of thickness is elusive, it is meant to signal a certain union between descriptive and evaluative content in a concept (Kirchin [2013]). For example, 'well-being' is thick to the extent that it is a good thing to have, but also to fare well is to have a certain amount of health, not to be depressed, lonely etc. ${ }^{5}$

Thick concepts generate a number of controversies especially in metaethics and philosophy of language. They have been thought to undermine the possibility of a moral theory (Williams [1985]), to expose the limits of the fact/value distinction (Putnam [2002]), and to create problems for cognitivism (Blackburn [2013]). Others disagree that thick concepts need to be that significant; indeed they might well be compatible with a number of metaethical views (Roberts [2013]).

My intention is to discuss mixed claims in science while inheriting as few of these foundational controversies as possible. Some philosophers take moral claims to express facts, others don't. Either remains an option for mixed claims. Those who take mixed claims literally will presumably treat thick concepts as referring to real entities with causal powers: for example, poverty, a phenomenon picked out by a thick concept, really does, on this view, have the power to cause heart disease. Those with more cautious metaethical views are free to adopt an antirealist reading of mixed claims instead: perhaps it is just a convenient manner of speaking to say that poverty causes heart disease. Either group should be interested in what I have to offer - ground rules for evaluating mixed claims in a scientific context.

\section{Mixed claims are different}

To formulate such rules I start by differentiating the value-ladenness of mixed claims from other kinds of value-ladenness. A taxonomy of the ways in which non-cognitive values can enter science is interesting in itself, but its more immediate purpose is to show the uniqueness of mixed claims. ${ }^{6}$

\subsection{Values as reasons to pursue science}

To value knowledge, both theoretical and applied, is to value understanding and perhaps also the possibility of control over the environment. Without this

\footnotetext{
${ }^{5}$ My mixedness is a property of claims rather than concepts, but if we were to extend the property of thickness to propositions and not just concepts, then mixed claims would plausibly come out as thick. 'Someone who is well does not cry herself to sleep' would be an example of a thick proposition. I will reserve the term 'mixed' for hypotheses and 'thick' for concepts.' Thick description' has a distinct meaning in philosophy of social science denoting ethnographic accounts that locate an event or a practice within a culture (Geertz [1973]). Since not all thick claims will amount to a thick description in the sense used in anthropology it is wise to keep the term 'mixed claims'.

${ }^{6}$ This taxonomy is a product of conversations with Stephen John.
} 
normative stance the pursuit of science as a social enterprise makes little sense. But this sense of value-ladenness clearly does not imply that individual scientific claims presuppose a specific standard about, in our case, well-being. It is entirely conceivable to value knowledge without pursuing mixed claims.

\subsection{Values as agenda-setters}

Normative commitments about what phenomena are interesting, important and worth studying partially set research agendas. For social sciences, Max Weber famously emphasized the role of cultural, moral and other commitments for selection of one ideal type over another (Weber [1949]). Nowadays a similar argument is made by several others and not just about the social sciences. Hugh Lacey ([1999], [2005]) identifies 'autonomy' as one of the senses of value freedom and defines it as the absence of external influence of moral, cultural and economic nature on the priorities and direction of basic research. He maintains that such autonomy is an impossible ideal, just because any scientific inquiry must start with a strategy that specifies what there is in the world to be known and how to proceed. Any such strategy is formulated from a cultural and historical standpoint and will prioritize some phenomena and methods over others by appeal to moral or cognitive values. A failure of autonomy, Lacey claims, need not necessarily compromise the authority of science. Philip Kitcher's ideal of a well-ordered science also calls for moral and political values endorsed by a representative community, to determine the agenda of scientific research (Kitcher [2011]).

Exactly how values should determine the agenda of science remains up for grabs. For our purposes, we only need to distinguish this agenda-setting function of values from their role in mixed claims. There can be moral and political reasons to initiate a scientific study of human and animal well-being, but these reasons alone do not force us to go mixed. We could instead insist on new value-free definitions of well-being as we shall see in Section 4.

\subsection{Values as ethical constraints on research protocols}

A third and probably the least controversial role for values is the specification of ethical constraints on scientific research. These constraints direct how to treat human and animal subjects during experiments, surveys and clinical trials. Again nothing here speaks for or against the use of normative categories to define the target phenomena as in the case of well-being research. A scientific protocol can be ethical or unethical irrespective of whether the claims it produces presuppose non-cognitive values.

\subsection{Values as arbiters between underdetermined theories}

When empirical evidence alone is insufficient to adjudicate between two or more theories, values have been noticed, indeed called, to close the gap. Feminist philosophers in particular have invoked this argument to point out the legitimate role in theory choice of moral and political values (Longino [1990], Kourany [2003] and many others). 
Our case is different. Take a mixed claim that long commutes are on average bad for well-being. This claim could, of course, be underdetermined by evidence. Is it really the commute? Maybe commuters are grim characters to start with? Confirming the badness of commuting for well-being requires a variety of intricate evidence: negative emotions, stress hormone levels, irritability, selfreports and behavior. Values, even non-cognitive ones, could undoubtedly enter to adjudicate between equally confirmed mixed hypotheses. But crucially for us, this process is distinct from the mixed case: in mixed claims, say about wellbeing, the standard of well-being itself is not what closes the gap.

\subsection{Values as determinants of standards of confirmation}

Another role for values explored originally in the 1950s by Richard Rudner and revived recently by Heather Douglas is in setting the level of evidence required for the acceptance of empirical hypotheses (Douglas [2009]). When there is uncertainty about a hypothesis that can inform important policy decisions (for instance, that drug X has certain side effects), moral considerations can be used to settle the level of evidence required for this hypothesis. Depending on the gravity of the consequences, a different level of evidence can be required. When the suspected side effect of the drug in question is as serious as a heart attack, a relatively small amount of evidence can be sufficient to accept the hypothesis 'Drug X causes heart attacks'.

There is still a debate about whether or not such an importation of values into science is legitimate (John [2015]). But regardless of the outcome, the fate of mixed claims remains unaffected. Mixed claims can take inductive risks just as much as non-mixed claims. They would still remain value-laden even if moral considerations were purged from decisions about the level of evidence required.

\subsection{Values as sources of wishful thinking and fraud}

The history of science is in many ways a story of non-cognitive values, along with fear, desire for glory and power, entering into production of knowledge. In our mixed cases, as we shall see in section 5.1, these values too can determine what claims are accepted. But there is a prima facie distinction between clear wrongs such as fudging data, falsifying results, or rejecting a theory because it's Jewish, on the one hand, and basing science on thick concepts as in our case. It may still turn out that mixed claims are illegitimate, but that should be for a different reason than the illegitimacy of wishful thinking and fraud.

This completes our taxonomy for present purposes. There are plausibly other roles for values in science, but the bottom line is that mixed claims are in a class of their own - they need to be discussed separately. Hugh Lacey's notion of 'neutrality' captures our case. According to neutrality, scientific claims neither presuppose nor support non-cognitive value judgments (Lacey [2005], pp. 2526). ${ }^{7}$ Mixed claims clearly violate neutrality. In the science of well-being in

\footnotetext{
${ }^{7}$ Lacey eventually redefines neutrality as inclusiveness and evenhandedness (Lacey [2013]), an ideal which mixed claims can satisfy as we shall see in sections 6 and 7.
} 
particular, mixed hypotheses presuppose a given standard of well-being, and in doing so can favor some conception of prudential value over another.

Now we can ask the big question: Are mixed claims legitimate in science?

\section{Mixed claims should stay}

The most explicit case against mixed claims can be found in Ernst Nagel's classic The Structure of Science, in a section entitled 'On the Value-Oriented Bias of Social Inquiry'. In it Nagel discusses the possibility that social science cannot, even in principle, be value free. He cites Leo Strauss's examples of quintessential thick concepts - art, religion, cruelty - agreeing that the evaluative content is there and that it may be practically difficult to extricate it from the positive content. However, it is still possible if we distinguish between two senses of value judgment at play: one 'appraising' and the other 'estimating' (Nagel [1961], pp. 492-493). We appraise when we endorse an ideal and judge something as meeting it or failing to meet it. We estimate when we judge to what extent a given phenomenon exhibits the features characteristic of a given ideal. Nagel's example is of anemia, but I shall apply his distinction to well-being. Social scientists appraise when they take a stance on what well-being is and then use it to judge whether a person or a community is doing well. On the other hand, they estimate, when, using an account of well-being, they judge how much a person or a community exhibit the features this account deems as well-being constitutive. In the first case, there is a genuine value judgment, while in the second a mere use of a normative criterion to make an empirical claim.

Nagel's goal in that chapter is a narrow one - only to establish that there is nothing inherently different about social sciences in the way they use values. For that, Nagel points out that physicists and biologists would also face the same issues when working with notions of 'efficiency' and 'anemia'. I readily agree. But his prising apart of appraisal from estimation has more ambitious intentions. The point of drawing the distinction is to eliminate appraisal from science, leaving only estimation. The ideal science for him is an ethically neutral one (Nagel [1961], p.495). What I have called mixed claims are plausibly appraising claims on Nagel's picture. So his proposal, which has a long history ${ }^{8}$, would be to reformulate them as estimation claims and eliminate the appraisal element. How?

A natural way to implement Nagel's proposal is to convert mixed claims from regular causal or correlational claims into conditional claims. Take one of our

\footnotetext{
${ }^{8}$ John Stuart Mill endorses this ideal in his System of Logic: "A scientific observer or reasoner, merely as such, is not an adviser for practice. His part is only to show that certain consequences follow from certain causes, and that to obtain certain ends, certain means are the most effectual. Whether the ends themselves are such as ought to be pursued, and if so, in what cases and to how great a length, it is no part of his business as a cultivator of science to decide, and science alone will never qualify him for the decision." (Mill [1882], n.pag.).
} 
earlier examples: psychologist Jane Gruber's claim that happiness is not always conducive to well-being (Gruber et al [2011]). Gruber documents the negative effects of positive emotions on problem solving, social bonds, mental health etc. The title of her article - 'A Dark Side of Happiness? How, When, and Why Happiness Is Not Always Good' - reads very much as an appraisal claim. But we can reformulate it as an estimation claim as follows:

'If well-being is understood as good functioning across many domains and over the course of a life, then happiness can impede well-being.'

Since there is no commitment to the truth of the antecedent, this claim is value free in the sense of Nagel's estimation claims. Nagel's position can then be summarized as follows:

For any mixed claim involving a cause or a correlation $C$, a thick concept $T$ and an operationalization $O$ of $T$

1) Scientists can investigate estimation claims: 'If $T$ is operationalized as $O$, then $C^{\prime}$.

2) Scientists cannot investigate appraisal claims that have not been so conditionalized.

\subsection{Against Nagel}

Nagel's proposal eliminates values at one stage, but it only pushes them to another less appropriate stage, as I shall argue now.

Suppose we went with Nagel and reformulated mixed claims into estimation claims, there would still remain a question as to which normative standard scientists should use in their estimation claims. What operationalization should Gruber use in the antecedent? I can think of three answers a Nagelian could give.

The first one is to recommend that scientists stick to the proverbial folk theory of well-being. More generally mixed claims could be rendered value-free if they defined their thick concepts using the value judgments of the community they studied. 'Happiness can impede that which our community calls well-being', would be Gruber's claim. Or when both the putative cause and the putative effect are thick we get the following: 'What our community calls secure attachment is a major cause of what our community calls child well-being'. The problem with this proposal is that the folk disagree even within one community and any proposal for how such a disagreement can be resolved is itself normative.

The second Nagelian proposal is to counsel that scientists study the empirical relations between well-being and a given factor on all the existing views of wellbeing. If these are fairly represented by hedonic, life satisfaction and flourishing approaches in psychology, then the science of well-being should build up a store of conditional claims: 
'If well-being is positive hedonic profile, then it is caused by...'

'If well-being is life satisfaction, then it is caused by...'

'If well-being is a sense of flourishing, then it is caused by...'

But it is hard to see why we should stop at these three. History of philosophy especially if we look beyond the Western traditions, boasts of other theories of well-being: well-being as knowledge of God, well-being as a meditative state, etc. Using them all is impossible, but a choice requires a normative judgment about their relative plausibility - a judgment that the Nagelian hopes to keep out of science.

The third and most likely Nagelian proposal is some sort of division of labor: scientists take care of facts, while others take care of values. The Nagelian would presumably argue that the right standard of well-being to use in the science of well-being is within the purview of moral philosophers (or also democratic decision makers). Scientists can participate in this discussion, but not qua scientists.

This proposal should also be rejected. In mixed cases normative decisions do not occur just at the beginning of the scientific process when the object of study is defined. Rather they keep reoccurring throughout, all the way down to the many practical decisions of scientific protocol. Those who define well-being in terms of authentic happiness, need an account of authenticity and a whole string of other value-driven notions about how to measure it properly (Sumner [1996], Tiberius [2013]). The economists adhering to the preference view of well-being refer to the notion of 'clean' preferences to differentiate authoritative from unauthoritative desires (Benjamin et al [2014]). Definitions of child well-being refer to healthy and unhealthy parental involvement. When divorce is viewed as a transformation rather than only as a loss, it is worth studying the evolution of divorcees' coping strategies long after the divorce and not just their shock and loneliness immediately after (Anderson [2004]). And so on and so forth. On the separation picture, the scientist keeps running back and forth to the philosopher (or keeps changing her identify from scientist to philosopher) whenever an evaluative question arises.

It is not the impracticality of this proposal that offends. After all ethicists (or other specialists on thick concepts) could, on Nagel's proposal, be 'embedded' in a scientific process, for example as members of the lab who step in to make a normative judgment. Rather the problem with the proposed division of labor is that it ignores or devalues scientists' knowledge about values, which they have acquired in virtue of their knowledge of facts. This knowledge enables them to make better normative choices qua scientists. It is because developmental psychologists know the effect of, say, institutionalization of orphans that they believe attachment to be crucial to child well-being. Similarly, it is because divorce scholars know the consequences of divorce that they conceptualize it as an opportunity for personal growth and not merely a loss (Anderson [2004]). In all these familiar cases value judgments are a result of an epistemic process, they are informed in part by facts and in part by the earlier value judgments made to detect those facts. Because of this process of co-evolution, scientists are in a good 
position to make some value judgments. Consulting philosophers and the public when making normative choices is important, but that does not mean scientists should refrain from using their own hard earned normative knowledge. ${ }^{9}$

So the Nagelian division of labor ignores the methodological realities of mixed sciences and wastes the normative knowledge scientists acquire while in the business of producing mixed claims. That much is sufficient for a prima facie case that mixed claims are worth preserving.

\section{The Dangers of Mixed Claims}

What if mixed claims, defensible in theory, are dangerous in practice? They might, for instance, bring with them dogmatism, bias and wishful thinking. These are the very charges that have been levied against proposals of feminist science (Pinnick et al [2003]) and that advocates of feminist science have gone to lengths to deny (Anderson [2006] among others).

It is an empirical question to what extent mixed claims, as compared to nonmixed ones, foster scientific malpractice. There is no data on whether mixed claims are treated more or less dogmatically, or whether its proponents are more or less likely to engage in wishful thinking. Here I allow for this possibility, but rather than speculating I concentrate straightaway on two well-documented dangers specific to well-being science.

The most serious charge is an importation into a science of substantive views about the nature of well-being that those whose well-being is being studied have good reasons to reject. When eminent economists including Nobel Prize winners advocate a measure of national well-being that takes into account only the average ratio of positive to negative emotions of the populace (Kahneman et al [2004a]), the citizens can legitimately object if they take well-being to consist in more than that. Perhaps they believe that national well-being should also encompass the compassion, kindness, mutual trust of their community, or the sustainability of their lifestyle, not to mention justice.

A related danger is when the scientists engaged in mixed science fail to notice the value judgments they are making. Economists have been known for treating preference satisfaction as revealed by willingness to pay as definitional of well-

\footnotetext{
${ }^{9}$ To treat values as responsive to facts commits no metaethical sins. As Anderson points out, even if Hume's prohibition of inferring facts from values is correct, values can still be supported by facts: 'Even if we grant that no substantive value judgment logically follows from any conjunction of factual statements, this merely puts value judgments on a logical par with scientific hypotheses. For it is equally true that there is no deductively valid inference from statements of evidence alone to theoretical statements. Theories always logically go beyond the evidence adduced in support of them. The question of neutrality is not whether factual judgments logically entail value judgments, but whether they can stand in evidentiary relations to them' (Anderson [2004],p.5)
} 
being and thus not needing a justification. 'Cost-benefit analysis is what evaluation means!' said a UK Treasury economist to a Whitehall civil servant I talked to recently. In those cases presenting empirical findings about well-being, freedom or health while failing to make explicit the normative assumptions on which these findings depend, amounts to misusing the authority of science. It sneaks controversial values in through inattention.

Let us call these dangers 'imposition' and 'inattention' respectively. They are not the only dangers, but I submit they are the most visible and distinctive of wellbeing science. They undermine trust in it and raise the danger of coercive paternalism. ${ }^{10}$ But instead of banning mixed claims from science, I propose to look for principles for their use that, though they may not guard against every danger, would at the very least guard against these two mistakes.

\section{The Existing Accounts of Objectivity}

A natural place to look for such principles is in the accounts of scientific objectivity friendly to values. As we shall here they are of limited help.

Perhaps the best known such account is Helen Longino's, summarized by herself thus:

Data (measurements, observations, experimental results) acquire evidential relevance for hypotheses only in the context of background assumptions. These acquire stability and legitimacy through surviving criticism. Justificatory practices must therefore include not only the testing of hypotheses against data, but the subjection of background assumptions (and reasoning and data) to criticism from a variety of perspectives (Longino [2008], p.80).

She argues that this sort of criticism can be secured by a community characterized by the following features: availability of venues for criticism, uptake of criticism, public standards to which theories and procedures can be held, and an equality of intellectual authority (Longino [1990],pp.76-79, among other places). Like Longino, Hugh Lacey too emphasizes pluralism of research strategies as a way of counterbalancing the value-ladenness of background assumptions (Lacey [2005]). When scientific research proceeds from multiple ideological and metaphysical stances and when each is forced to justify itself in a public forum, the outcome is an objective inquiry, so the story goes. Douglas [2004] aptly calls this 'interactive objectivity'. I think interactive objectivity is not enough.

\footnotetext{
10 For an argument to this effect see Haybron and Alexandrova 2013 where we define the notion of 'inattentive paternalism'.
} 
Pluralism and open criticism need a more robust formulation specific to the case of mixed claims. Otherwise these criteria are too vague for guarding against imposition and inattention.

Pluralism about definitions of well-being already characterizes the science of well-being. No single definition of well-being dominates the current landscape. Psychology alone boasts three as we have seen; health sciences use quality of life adapted to different diseases; developmental psychology has a conception of child well-being; mainstream economics is wedded to preferences as revealed by choices; development economics uses an objective quality of life approach, and so on and so forth (Alexandrova [2012]).

Such a variety of definitions could alert researchers to the problems of inattention and imposition. But by itself pluralism does not ensure that moral presuppositions are noticed and scrutinized in the right way. Measures of wellbeing are often selected for their ease of use, psychometric properties, institutional and disciplinary inertia, or personal preference. There is no guarantee in pluralism alone that these choices are noticed and called out for imposition and inattention. It is also not enough to say, as Longino and Lacey do, that different research programs need to be open to effective criticism. Mixed claims need a very specific sort of criticism on normative grounds, not just any criticism.

\subsection{The perils of impartiality}

Another common criterion of objectivity - 'impartiality' - faces a different problem: on one formulation it excludes mixed claims outright, on another it allows for mixed claims but without helping with inattention and imposition. ${ }^{11}$ Impartiality specifies that cognitive values alone, and not moral and political ones, should justify our acceptance and rejection of theories (Lacey [2005], pp.23-24). To violate impartiality, it is claimed, is to commit the error of wishful thinking. Speaking of social sciences in particular Douglas argues:

If values [...] serve as the reason in themselves for a theory choice, we have confused the normative and the descriptive in precisely the ways that Weber and Nagel warned us against. Our values are not a good indication, in themselves, of the way the world is (Douglas [2011], pp.2324). ${ }^{12}$

The problem is that when impartiality is formulated in a standard way as forbidding that values determine our acceptance of hypotheses, mixed claims face a test they could not possibly pass. This is because in a mixed claim the

\footnotetext{
11 Impartiality is endorsed by Weber [1949], Douglas [2009], Lacey [2005] among others. 12 To be precise, Douglas's conception of impartiality is different from Lacey's. She does not rely on the distinction between cognitive and non-cognitive values, but instead on the distinction between direct and indirect roles of value. Once values have been invoked 'directly' in our choice of what to study and methodology, no further direct role of values is permitted. When it comes to confirmation of hypotheses values are only to be used 'indirectly' for managing uncertainty (Douglas [2009], chapter 5).
} 
initial value judgment does preclude certain findings and to this extent values do determine what we will find.

Consider an example: a staunch Aristotelian about well-being who believes that only the virtuous can flourish, includes a morality constraint in her measure of well-being (ignore for a moment the practical difficulty of doing so). Using this measure she finds that well-being is very low in a community of sociopaths. Clearly this finding is determined in part by her initial value judgment and in this sense it fails the impartiality test. But equally psychologists who use life satisfaction questionnaires cannot discover a well-faring albeit constantly complaining kvetch, while those who use purely hedonic measures cannot discover a well-faring tortured artist no matter how eagerly she endorses her life.

Definitions of well-being constrain the range of available findings, just as theories constrain the range of available observations. When a prudential value judgment is part of the background theory, impartiality thus defined cannot be sustained. It makes mixed claims come out illegitimate by definition. They cannot escape the company of wishful thinking and scientific fraud. This is unsatisfactory: the legitimacy of mixed claims should not be a matter of definition. A better formulation of impartiality is as follows:

Impartiality2: A mixed claim is impartial if and only if, once all the value decisions about the measures, methods and required levels of confirmation are made, non-cognitive values to do not play any further role in determining whether the hypothesis is accepted.

To be fair, this is probably the version closest to what advocates of impartiality have in mind ${ }^{13}$, and it may well be acceptable to them. All I claim is that Impartiality 2 does not help us with imposition and inattention. This rule guards against the imposition of values into claims already stripped of any values but epistemic. It cannot therefore tell us how to deal with claims that are not stripped of them, for example, mixed claims.

So while pluralism, open criticism and impartiality2 are important, perhaps necessary, mixed science needs additional principles.

\section{Objectivity for Mixed Claims}

The additional principles we are looking for are partly principles of objectivity about values - prudential, ethical, political, whichever feature in mixed claims.

\footnotetext{
${ }^{13}$ Lacey [2003] makes allowance for the use of values in methodological choices in the human sciences and Douglas would classify the choice of which thick concept to study as an initial methodological decision in which the direct use of values is permitted [personal conversation]. Anderson [2004] too is careful in formulating impartiality: "If a hypothesis is to be tested, the research design must leave open a fair possibility that evidence will disconfirm it" (p.19, my italics), the implication being that choices of methodology are not always meant to be tested.
} 
To trust a science of well-being is in part to trust that it is based on an appropriate conception of well-being. But objectivity means (and has meant ${ }^{14}$ ) many things; so before I state the principles that realize it for mixed claims, I distinguish my focus from other objectivities.

Janack [2002] identifies no fewer than twenty senses of objectivity in contemporary philosophy of science alone. More manageably Douglas [2004] draws a three-way distinction: (1) objectivity as a way of 'getting at the objects' as they really are, (2) objectivity as a way of minimizing bias, and (3) objectivity as a characteristic of the social process of science. ${ }^{15}$ Each of the three senses mark a legitimate goal for the science of well-being. Focusing on (1]), we might ask whether well-being is a plausible scientific object - is it stable enough to persist over peoples and histories enabling meaningful comparisons and theory building? Is it robust enough to changes in our instruments and methods? Focusing on (2), we might worry, as we already have in the discussion on impartiality, about dogmatism and wishful thinking. While both of these foci are eminently legitimate, they do not help with imposition and inattention. Securing the right normative assumptions for mixed claims is neither a metaphysical task of making sure well-being is out there, nor is it a task of eliminating values.

Rather I am after the sort of objectivity that ensures that values have undergone an appropriate social control, giving a community reasons to trust this knowledge. Such a control may not warrant blanket trust in a research project on the whole, but it would at least warrant trust in the project's value presuppositions and at least by the community that exercized control over these values. This sense of objectivity is closest to Douglas's (3) and to the 'procedural objectivity' that became popular in the twentieth century (Porter [1995], Fine [1998]). Procedural objectivity focuses on the process of inquiry, not its results, aiming to ensure that this process is transparent, legitimate and resistant to hijacking by specific individuals or groups.

Historically, procedural objectivity has been thought to require value-freedom understood as impersonality, i.e. procedures should not presuppose the point of view of any particular person, group, or ideology (ibid). But value-freedom and procedural objectivity do not stand or fall together. There could be good procedures for dealing with values. This is the conceit of philosophers who defend accounts of procedural moral objectivity inspired by the pragmatism of John Dewey. On this account the objective values are those that survive criticism in the public sphere and that are tested through 'experiments in living' (Putnam [2002], Anderson [2014], Brown [2013], Roth [2013]). This pragmatist story is often contrasted to constructivist conceptions of political objectivity that justify the principles of, say, a liberal democratic state by appeal to outcomes of a more or less ideal deliberation (Rawls [1996], Nussbaum [2001], Gaus [2011] among many others).

Scientists who put forward and test mixed claims do not have access to ideal deliberation. This fact of life favors the pragmatist story for our case. But rather

${ }^{14}$ See Daston [1992] on historical shifts in conceptions of objectivity.

15 Douglas [2004] also draws further distinctions within each of the three modes, but they do not all concern us. 
than entering the debate between pragmatists and ideal theorists, I move straight to those actionable principles that when used by a scientific community will deal with imposition and inattention. These principles are compelling whichever precise story about procedural objectivity is adopted.

\section{Three Rules}

\subsection{Unearth the value presuppositions in methods and measures.}

Inattention is a failure to acknowledge the values shaping a research agenda. Philosophers of science of all persuasions have urged making these presuppositions explicit (Weber [1949], Nagel [1961], Hausman and McPherson [2006], Douglas [2011] and others]. I agree that this is the first step. Depending on the case, explicitness is more or less straightforwardly implemented.

Sometimes all it takes is a sentence in the Methods section of a journal article: 'In this study we assume that well-being consists in a favorable ratio of positive to negative emotions'. At other times, when scientific formalisms hide the value presupposition, it takes a great deal of work, often a philosopher's eye ${ }^{16}$, to uncover them.

One obstacle to explicitness is the sheer absence of an underlying theory in some cases. For example, measures of quality of life are often indices constructed from several indicators. In the social indicators tradition, child well-being is measured by an index of infant mortality, vaccination, school attendance and a few other statistics (Land et al [2001]). No researcher pretends that these factors 'are' child well-being. They are only meant to be indicators of it. What then is child wellbeing? This question is usually left unanswered by the scientists averse to philosophizing. ${ }^{17}$ But if explicitness is needed to combat inattention, and if inattention is an obstacle to procedural objectivity, such a failure to philosophize about the nature of well-being is a failure of procedural objectivity. So whenever scientists measure or otherwise study the well-being of $X$, they should be able to say, at least in outline, what the well-being of $X$ is; otherwise they are not attending to their value presuppositions. In the next section I consider the case when the precise definition does not matter because the empirical relation of interest holds on 'any' definition. But even then scientists should say what accounts of well-being the different measures presuppose.

As well as laying cards on the table, explicitness calls for an acknowledgment of alternative presuppositions, or at least awareness that they exist and that the disagreement about them is in part a substantive disagreement about values and not just a difference about which measures are more convenient. Though this

\footnotetext{
${ }^{16}$ Hausman and McPherson [2006] chapter 2 is a classic illustration of how to unearth the moral assumptions in economic reasoning.

17 On the absence of a theory of child well-being and what to do about it see Raghavan and Alexandrova [2015].
} 
aspect might take some scientists out of their comfort zone, explicitness is realistic to achieve. But merely having values in the open does not guard against imposition. The next two rules show what to do when disagreements about values arise, for example when relevant parties differ in their conception of wellbeing.

\subsection{Check if value presuppositions are invariant to disagreements.}

Sometimes measures of well-being are 'robust' to fundamental philosophical disagreements. At their best, measures of child well-being, for example, attempt to capture conditions that if realized in childhood enable children to grow up happier, healthier, and more positively connected to others. Thus these measures stand up on all major of theories of well-being that contemporary Western community entertains - experiential, reflective and objective. At least this is the hope. It is this robustness - an invariance to several different conceptions of well-being - that gives some mixed claims objectivity on the cheap, so to speak.

If such a robustness exists, scientists can stop here. But philosophizing will not always be avoided so easily. Sometimes it matters a great deal which precise measure of well-being is selected. Today some governments want to measure and track national well-being (Stiglitz et al [2009], Office of National Statistics [2012]). There is no shortage of academic opinions on the matter. Daniel Kahneman and colleagues advocate a hedonic measure - a nation is doing well to the extent that its populace has on average a favorable balance of positive over negative emotions (Kahneman et al [2004a]). Development economists prefer measures based on objective quality of life (Nussbaum and Sen [1993], Dasgupta [2001]). Yet others work with a life satisfaction notion of well-being, which purports to best respect the judgment of the individual (Diener et al [2008]). But these different measures are not robust in the sense above. A 2010 article by Kahneman and Deaton has a self-explanatory title 'High income improves evaluation of life but not emotional well-being'. Depending on whether scientists use life satisfaction measures (which capture evaluation) or happiness measures (which capture emotional well-being), they will reach radically different verdicts on whether economic growth promotes well-being. Which measure is correct is a choice that in a democratic society should be made in a way that the next principle proposes.

\subsection{Consult the relevant parties.}

When the choice of a measure of well-being is a choice between conflicting sets of values, the only way to practice trustworthy science is to make this choice in a deliberative public setting in which the relevant parties are included. A measure of well-being that survives public scrutiny has procedural objectivity. Consider an example. 
Between 2010 and 2012 the UK Office for National Statistics (ONS) conducted a country-wide inquiry called "What matters to you?" (ONS [2012]). Potential measures of well-being were released publically with the following questions:

1. Do you think the proposed domains present a complete picture of wellbeing? If not, what would you do differently?

2. Do you think the scope of each of the proposed domains is correct? If not, please give details.

3. Is the balance between objective and subjective measures about right? Please give details.

The outcome of this exercise is a measure of UK's well-being that contains both subjective indicators - happiness, life satisfaction, sense of meaning - and also objective indicators, such as life expectancy and educational achievements. The ONS settled the seemingly intractable debates between the experts by including as many items into its final measure as practically possible, and also by having the public vet this measure. No doubt the ONS measure has problems, but the honest effort to canvass the diverse views shows that the value presuppositions on this measure have arguably passed the sort of test I have in mind.

This example combines features of two relatively recent experiments in political science and science studies respectively: deliberative polling and systematic participation of the public in science. Deliberative polling (Fishkin [2009]) occurs when a representative sample of the public comes together for a small group session with a moderator to discuss a question of public policy (for example, should a minimum wage be required?). The participants get input from the scholars who are experts on the topic via preliminary briefings. Moderators are trained to foster a respectful and inclusive debate. At the end the attitudes of the participants are measured and compared against their earlier attitudes. While political scientists find ways of building consensus about politics, science studies scholars explore ways for people affected by a piece of science or medicine to have a systematic and non-trivial say in its methods, assumptions or applications, while at the same time respecting existing scientific expertise. ${ }^{18}$

Putting these two traditions together I propose 'deliberative polls of normative presuppositions of mixed claims'. Groups of deliberators could be presented with various options for conceptualizing well-being (or freedom, health, etc.), and with the relative advantages of each option normatively and practically. The deliberators will attempt to reach agreement according to whatever consensusbuilding and voting rules they decide to put in place. Even if not everybody favors the values that survive such an exercise, the resulting consensus has some legitimacy and deserves trust at least from those whose views are admissible in a democracy and have been heard.

Such deliberations should include samples of all concerned parties. The ONS consultation happened by soliciting responses to an online questionnaire widely advertised through the ONS website, letters and public events. Generalizing from this example, I suggest that for mixed claims about well-being the deliberative

${ }^{18}$ Chilvers [2008] and Douglas [2005] provide an overview of the history and the recent efforts. 
polling should include (a) the scholars of different approaches to well-being (plausibly philosophers or anthropologists), (b) the researchers doing the measurement and data collection, (c) the policy users of this knowledge, and (d) a representative sample of the subjects who are likely to be affected when this knowledge is put into practice through policy, therapies and other interventions.

The inclusion of experts is important because as I argued in 4.1, scientists have normative knowledge in virtue of their empirical knowledge. Respecting this knowledge means that decisions about measures of well-being should not be outsourced to the non-scientists. But including the non-scientists is no less important. When scientists measure and monitor well-being this information can be used for oppression and domination. Science after all has the power and indeed a well-documented tendency to devalue non-expert sources of knowledge. ${ }^{19}$ Having people weigh in on how their own well-being is measured is a prudent reaction to these dangers, a reaction that need not assume that wellbeing is whatever people say it is. ${ }^{20}$

Can such exercises respect the expertise of scientists on well-being while at the same time avoiding imposing values on non-scientists? This depends on the implementation: what proportion of scientists to non-scientists is in the group, how the final consensus is determined, what checks there are on power imbalances. These are hard but not intractable questions. The deliberative exercises I sketch here are expensive, difficult to realize and uncertain in their fruitfulness. So it is an open question whether mine is a rational bet all things considered. But it seems wrong not to try.

\section{Conclusion}

I proposed three rules: to make explicit the value presuppositions of mixed claims, to check whether the empirical claim is robust to disagreements about values, and finally, if it isn't robust, to expose these values to an inclusive deliberation.

Together these three principles ensure that the science of well-being neither imposes values, nor sneaks them past the people whose well-being is in question. Following these rules, I submit, secures procedural objectivity for the value presuppositions of this science. For other mixed claims, such as about freedom or health, these principles may need to be amended, but the spirit - objectivity as open vetting - should remain the same.

When the very definition and measurement of phenomena depends on moral categories, as they do in mixed sciences, we face a choice. We could reserve the notion of objectivity only for decisions and practices that avoid any such values.

${ }^{19}$ Wynne [1989] is a classic study of this phenomenon.

20 See Haybron and Tiberius [2015] for an argument that in a policy context well-being measures should be sensitive to the priorities of the citizens whose well-being is in question. 
This is a view that preserves the neutrality of science at the expense of expelling mixed claims. I have argued against this. Mixed claims are already part and parcel of science. Pretending that they can be reformulated into value-free claims devalues perfectly good knowledge and stakes the authority of science on its separation from the community that enables it. The alternative I favor is to broaden our notion of objectivity to encompass also value-based decisions, such as which measures of well-being to adopt and when.

\section{Acknowledgements}

For valuable comments and advice I thank three anonymous referees, Stephen John, Tim Lewens, Adrian Boutel, Valerie Tiberius, Heather Douglas, Elizabeth Anderson, Mary Morgan, Markus Anderljung, Clare Chambers, Tom Dougherty, Bernardo Zacka, Paul Sagar and Robert Northcott.

Anna Alexandrova
Aa686@cam.ac.uk
Department of History and Philosophy of Science
University of Cambridge
Free School Lane
Cambridge
CB2 $3 R H$
United Kingdom

\section{Works Cited}

Alexandrova, A. [2012]: 'Well-Being as an Object of Science.' Philosophy of Science, 79[5], pp.678-89.

Anderson, E. [2014]: 'Social Movements, Experiments in Living, and Moral Progress: Case Studies from Britain's Abolition of Slavery.' Lindley Lecture Series, University of Kansas.

Anderson, E. [2004]: 'Uses of value judgments in science: A general argument, with lessons from a case study of feminist research on divorce.' Hypatia, 19[1], pp.1-24.

Anderson, E. [2006]: 'How not to criticize feminist epistemology: a review of Scrutinizing feminist epistemology.' <http://www-personal. umich. edu/\{ $\sim\{\}\}$ eandersn/hownotreview. html.>

Beddington, J., C.L. Cooper, J. Field, U. Goswami, F.A. Huppert, R. Jenkins, H.S. Jones, T.B.L. Kirkwood, B.J. Sahakian, and S.M. Thomas. [2008]: 'The mental wealth of nations.' Nature 455(7216), pp.1057-60.

Benjamin, D. J., Heffetz, O., Kimball, M. S., and Szembrot, N. [2014]: 'Beyond happiness and satisfaction: toward well-being indices based on stated preference.' American Economic Review, 104[9], pp.2698-735.

Blackburn, S. [2013]: 'Disentangling Disentangling.' In Kirchin 2013, pp.121-35.

Brown, M. J. [2013]: 'Values in Science beyond Underdetermination and Inductive Risk.' Philosophy of science, 80[5], pp.829-39. 
Chilvers, J. [2008]: 'Deliberating Competence Theoretical and Practitioner Perspectives on Effective Participatory Appraisal Practice.' Science, Technology \& Human Values, 33[2], pp.155-85.

Daston, L. [1992]: 'Objectivity and the escape from perspective.' Social studies of science, 22 [4], pp.597-618.

Diener, E., R. Lucas, U. Schimmack and J. Helliwell. [2008]: Well-being for Public Policy. New York: Oxford University Press.

Douglas, H. [2004]: 'The irreducible complexity of objectivity.' Synthese, 138[3], pp.453-73.

Douglas, H. [2005]: 'Inserting the Public into Science' in Democratization of Expertise? Exploring Novel Forms of Scientific Advice in Political DecisionMaking, Sociology of the Sciences vol. 24, Sabine Maasen and Peter Weingart [eds.], Springer, pp.153-69.

Douglas, H., [2009]: Science, Policy, and the Value-Free Ideal. Pittsburgh: University of Pittsburgh Press.

Douglas, H., [2011]: 'Facts, Values, and Objectivity', in Jarvie, I., and Zamora Bonilla, J., [eds.],The SAGE Handbook of Philosophy of Social Science, pp.513-29. London: SAGE Publications.

Dupré, J. [2007]: 'Fact and value', in H. Kincaid, J. Dupré and A. Wylie [eds] ValueFree Science? Ideals and Illusions. New York: Oxford University Press. Pp.2741.

Fine, A. [1998]: 'The viewpoint of no-one in particular.' In Proceedings and addresses of the American Philosophical Association. 72(2), pp. 7-20.

Fishkin, J. [2009]: When the people speak: Deliberative democracy and public consultation. Oxford: Oxford University Press.

Fredrickson, B.L. [2001]: 'The role of positive emotions in positive psychology: The broaden-and-build theory of positive emotions.' American psychologist, 56[3], pp.218-26.

Gaus, G. [2011]: The Order of Public Reason: A Theory of Freedom and Morality in a Diverse and Bounded World, Cambridge: Cambridge University Press.

Geertz, C. [1973]: 'Thick Description: Toward an Interpretive Theory of Culture' in The Interpretation of Cultures New York: Basic Books.

Gruber, J., Mauss, I.B. and Tamir, M. [2011]: 'A dark side of happiness? How, when, and why happiness is not always good.' Perspectives on Psychological Science, 6[3], pp.222-33.

Hausman, D. and M. McPherson [2006]: Economic Analysis, Moral Philosophy, and Public Policy. New York: Cambridge University Press.

Haybron, D.M. and Alexandrova, A. [2013]: 'Paternalism in economics.' In [eds] C. Coons and M. Weber Paternalism: Theory and practice, Cambridge, Cambridge University Press, pp.157-77.

Hawthorne, S. [2013]: Accidental Intolerance: How We Stigmatize ADHD and how We Can Stop. New York: Oxford University Press.

Haybron, D. and V. Tiberius [2015]: 'Well-being Policy: What Standard of Wellbeing?', forthcoming in the Journal of American Philosophical Association.

Janack, M. [2002]: 'Dilemmas of objectivity.' Social Epistemology, 16(3), pp.26781.

John, S. [2015]: 'The example of the IPCC does not vindicate the Value Free Ideal: a reply to Gregor Betz.' European Journal for Philosophy of Science 5(1), pp.113. 
Kahneman, D., A. Krueger, D. Schkade, N. Schwartz and A. Stone. [2004a]: 'Toward National Well-Being Accounts'. American Economic Review 94(2), pp.429-34.

Kahneman, D. and Deaton, A. [2010]: 'High income improves evaluation of life but not emotional well-being.' Proceedings of the National Academy of Sciences, 107[38], pp.16489-93.

Kahneman, D., A.B. Krueger, D.A. Schkade, N. Schwarz, and A.A. Stone. [2004b]: 'A survey method for characterizing daily life experience: The day reconstruction method.' Science 306(5702), pp.1776-80.

Kirchin, S. [Ed.] [2013]: Thick Concepts. Oxford, Oxford University Press.

Kitcher, P. [2011]: Science in a Democratic Society. Prometheus Books.

Kourany, J.A. [2003]: 'A Philosophy of Science for the Twenty-First Century.' Philosophy of science, 70[1], pp.1-14.

Lacey, H. [2003]: 'The behavioral scientist qua scientist makes value judgments.' Behavior and Philosophy, 31, pp.209-23.

Lacey, H. [2005]: Values and objectivity in science: the current controversy about transgenic crops. Lexington books.

Lacey, H. [2013]: 'Rehabilitating neutrality.' Philosophical studies, 163[1], pp.7783.

Land, K.C., Lamb, V.L. and Mustillo, S.K. [2001]: 'Child and youth well-being in the United States, 1975-1998: Some findings from a new index.' Social Indicators Research, 56[3], pp.241-318.

Longino, H.E. [1990]: Science as Social Knowledge: Values and Objectivity in Scientific Inquiry. Princeton: Princeton University Press.

Longino, H.E. [2008]: 'Values, Heuristics, and the Politics of Knowledge'. in The Challenge of the Social and the Pressure of Practice: Science and Values Revisited. Eds. M. Carrier, D. Howard, J. Kourany. Pittsburgh: University of Pittsburgh Press,pp.68-85.

Longino, H.E. [2013].:Studying human behavior: how scientists investigate aggression and sexuality. University of Chicago Press.

Mill, J.S. [1882]: A System of Logic, Ratiocinative and Inductive. Project Gutenberg.

Nagel, E. [1961]: The Structure of Science: Problems in the Logic of Scientific Explanation. New York: Harcourt, Brace \& World, Inc.

Nussbaum, M.C. [2001]: "Political objectivity". New Literary History, 32 [4], pp.883-906.

Office of National Statistics [2012]: 'Proposed Domain and Headline Indicators for Measuring National Well-being' Accessed on January $5^{\text {th }} 2015$. http://www.ons.gov.uk/ons/about-ons/getinvolved/consultations/archived-consultations/2012/measuring-nationalwell-being-domains/index.html

Pinnick, C.L., Koertge, N., and Almeder, R.F. [Eds.]. [2003]: Scrutinizing feminist epistemology: An examination of gender in science. Rutgers University Press.

Porter, T. [1995]: Trust in Numbers: The Pursuit of Objectivity in Science and Public Life. Princeton, NJ: Princeton University Press.

Putnam, H. [2002]: The Collapse of the Fact/Value Dichotomy and Other Essays. Cambridge, Mass: Harvard University Press.

Raghavan, R. and Alexandrova, A. [2015]: 'Toward a Theory of Child Well-Being.' Social Indicators Research, 121:3, pp.887-902.

Rawls, J [1993]: Political Liberalism. Columbia University Press. 
Reiss, J. [2010]: Error in economics: Towards a more evidence-based methodology. Routledge.

Roberts, D. [2013]: 'Thick Concepts.' Philosophy Compass, 8, pp.677-88.

Root, M. [2007]: 'Social problems', in H. Kincaid, J. Dupré, and A. Wylie [eds] Value-Free Science? Ideals and Illusions. New York: Oxford University Press. pp.42-57.

Roth, A.[2013]: 'A Procedural, Pragmatist Account of Ethical Objectivity.' Kennedy Institute of Ethics Journal 23 [2], pp.169-200.

Ryan, R.M., \& Deci, E.L. [2001]: 'On happiness and human potentials: A review of research on hedonic and eudaimonic well-being.' Annual review of psychology, 52[1], pp.141-66.

Sumner, L.W. [1996]: Welfare, happiness, and ethics. Oxford University Press.

Tiberius, V. [2004]: Cultural differences and philosophical accounts of wellbeing. Journal of Happiness Studies, 5.3, pp.293-314.

Tiberius, V. [2013]: 'Well-being, Wisdom and Thick Theorizing: On the division of Labour between moral philosophy and positive psychology'. in Kirchin 2013 (ed), pp.217-34.

Weber, M. [1949]: The Methodology of the Social Sciences [E.A. Shils and H.A. Finch, trans.]. Glencoe, IL: Free Press.

Williams, B. [1985]: Ethics and the Limits of Philosophy. Mass: Harvard University Press.

World Health Organization [1948]: Constitution. World Health Organization, Geneva. http://whqlibdoc.who.int/hist/official_records/constitution.pdf

Wynne, B. [1989]: 'Sheepfarming after Chernobyl: A case study in communicating scientific information.' Environment: Science and Policy for Sustainable Development, 31[2], pp.10-39.

Zacks, J.M. and Maley, C.J. [2007]: 'What's Hot in Psychology?' APS Observer, 20, pp.23-6. 